\title{
HYDRAENIDAE ET ELMIDAE DU PORTUGAL (COLEOPTERA)
}

\author{
par C. BERTHELEMY ${ }^{1}$ et L. S. WHYTTON DA TERRA ${ }^{2}$.
}

\begin{abstract}
Environ 1200 exemplaires d'Hydraenidae et d'Elmidae, appartenant à 30 espèces, ont été récoltés dans le Nord du Portugal, principalement dans des ruisseaux et des petites rivières coulant sur du granite, entre 30 et $935 \mathrm{~m}$ d'altitude. Presque tous les Hydraenidae sont des endémiques ibériques, y compris une nouvelle espèce d'Haenydra, $H$. lusitana nov. sp. Quelques Elmidae intéressants, tels que Limnius perrisi mediocarinatus, Oulimnius bertrandi et o. major ont également été capturés.
\end{abstract}

\section{Hydraenidae and Elmidae from Portugal (Coleoptera).}

About 1200 specimens of Hydraenidae and Elmidae, belonging to 30 species, were collected in Northern Portugal, mainly in streams and small rivers flowing on granite, between 30 and $935 \mathrm{~m}$ a.s.I. Almost all Hydraenidae are Iberian endemics, including a new species of Haenydra, $H$. lusitana nov. sp. Some interesting Elmidae, such as Limnius perrisi mediocarinatus, Oulimnius bertrandi and $O$. major were also found.

L'un de nous (W. d. T.) a récolté plus de 1200 Hydraenidae et Elmidae au cours de ses recherches sur les Ephéméroptères, Plécoptères et Trichoptères du Portugal. Presque toutes les stations sont situées dans le Nord du pays, entre les rivières Douro et Minho. Les récoltes effectuées au sud du Douro ont été tout à fait sporadiques (fig. 1).

Bien que les prélèvements n'aient pas été planifiés dans le temps et l'espace, l'étude de ce matériel a révélé des faits nouveaux. En effet, la répartition des Coléoptères dans les cours d'eau de la Péninsule Ibérique n'est encore connue que de façon fragmentaire (d'Orchymont 1936 a, b ; Berthélemy 1962, 1964 a, b, 1965 ; Bertrand i965; Olmi 1970).

1. Laboratoire d'Hydrobiologie, Université Toulouse III, 118, route de Narbonne, 31077 Toulouse Cedex, France.

2. Estaçâo Aquícola, Vila do Conde, Portugal. 


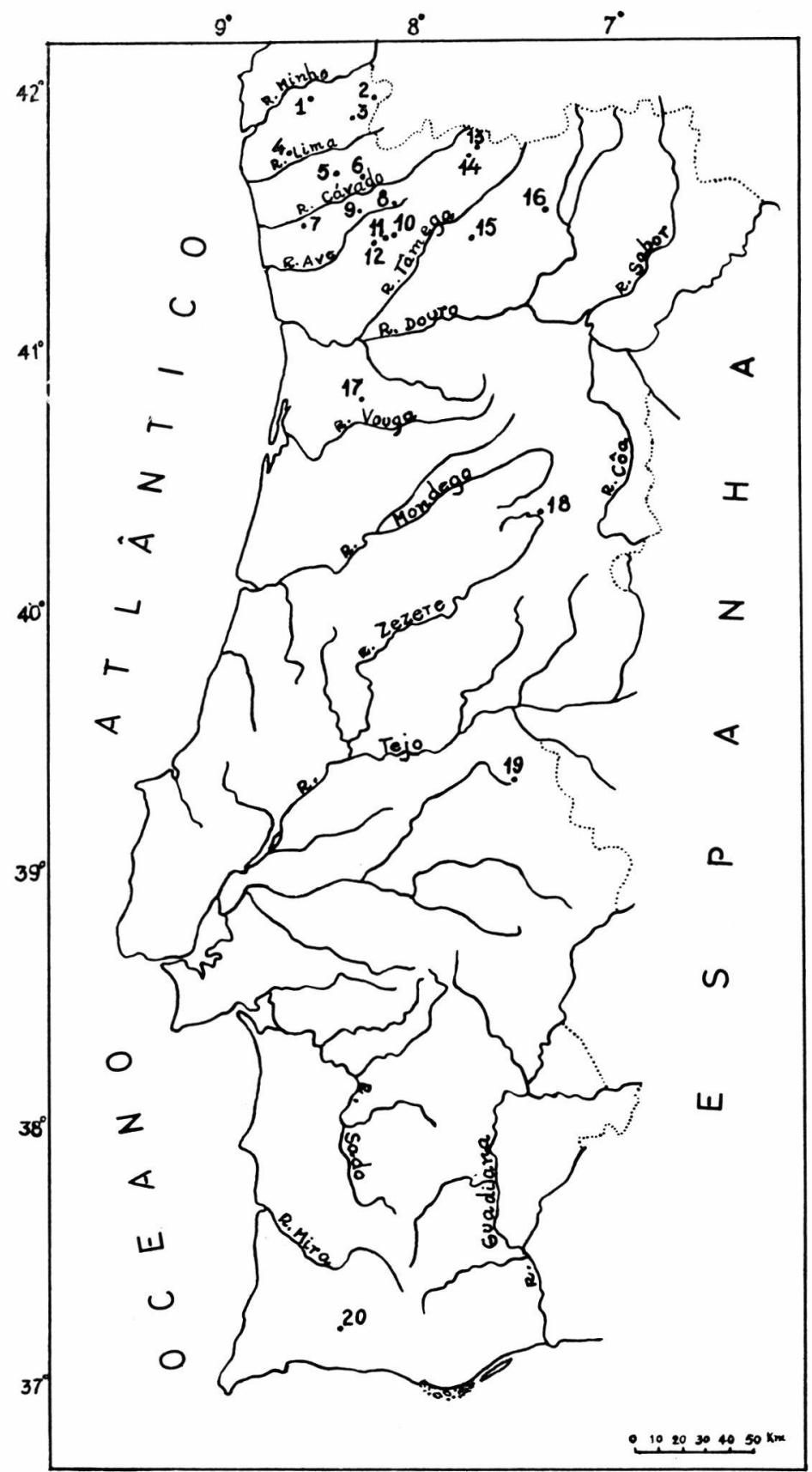

Fig. 1. - Répartition des stations prospectées 


\section{1. - LES STATIONS ET LEUR FAUNe}

Nous indiquons pour chaque station la latitude, la longitude, l'altitude (A), la pente (P), la largeur (1), la profondeur ( $\mathrm{pr}$ ), la nature du fond, les terrains traversés, l'aspect des rives et la température de l'eau au moment des prélèvements.

Station 1. - Rio Manco, à Gondomil.

$42^{\circ} \cdot 1^{\prime} 16^{\prime \prime} \mathrm{N}-8^{\circ} 33^{\prime} 6^{\prime \prime}$ W. A : $55 \mathrm{~m} ; \mathrm{P}: 10 \% ; 1: 1,5$ à $2 \mathrm{~m} ; \mathrm{pr}: 30$ à $40 \mathrm{~cm}$. Ruisseau lent; fond de pierres et de sable, avec quelques mousses; granite; terres de culture avec murets de support sur les deux rives; $16^{\circ} \mathrm{C}$ le 1:10.1968.

Station 2. - Rio Castro-Laboreiro, à Castro-Laboreiro.

$42^{\circ} 11^{\prime} 54^{\prime \prime} \mathrm{N}-8^{\circ} 9^{\prime} 20^{\prime \prime} \mathrm{W}$. A : $935 \mathrm{~m} ; \mathrm{P}: 9,5 \%$; 1:2 à $4 \mathrm{~m} ; \mathrm{pr}: 50$ à $120 \mathrm{~cm}$ (petit barrage en aval). Rivière de montagne à courant rapide; petits blocs, graviers et sable, mousses abondantes; granite; prairie bordée d'arbres sur une rive, brousse sur l'autre; $7^{\circ} \mathrm{C}$ le 13.10 .1972 .

Station 3. - Rio Vez, à Sistelo.

$41^{\circ} 58^{\prime} 23^{\prime \prime} \mathrm{N}-8^{\circ} 22^{\prime} 14^{\prime \prime} \mathrm{W}$. A : $230 \mathrm{~m} ;$ P. $27,5 \% ; 1: 3$ à $4 \mathrm{~m} ;$ pr. $: 50$ à $70 \mathrm{~cm}$. Rivière de montagne à courant rapide; fond rocheux avec blocs et gros galets; granite; vallée profonde $: 9,5^{\circ} \mathrm{C}$ le 23.10 .1972 .

Station 4. - Ribeira de Nogueira, à Nogueria.

$41^{\circ} 44^{\prime} 25^{\prime \prime} \mathrm{N}-8^{\circ} 43^{\prime} 55^{\prime \prime}$ W. A : $30 \mathrm{~m} ; \mathrm{P}: 27,5 \%$; $1: 80$ à $100 \mathrm{~cm} ;$ pr: 20 à $30 \mathrm{~cm}$. Ruisseau à courant modéré; graviers, vase; schistes et granite; prélèvement le 15.10.1968.

Station 5. - Rio Neiva, à Pedregais.

$41^{\circ} 41^{\prime} 48^{\prime \prime} \mathrm{N}-8^{\circ} 28^{\prime} 21^{\prime \prime} \mathrm{W}$. A : $240 \mathrm{~m} ; \mathrm{P}: 14,5 \%$; $1: 2$ à $3 \mathrm{~m} ; \mathrm{pr}: 30$ à $40 \mathrm{~cm}$. Partie amont d'une petite rivière à courant modéré; graviers, sable, végétation aquatique avec surtout des Oenanthe crocata L. et des mousses; granite; terres de culture avec murets de support sur les deux rives; $17^{\circ} \mathrm{C}$ le 1.7.1968.

Station 6. - Ribeira de Casaldelo, à Oriz.

$41^{\circ} 42^{\prime} 0^{\prime \prime} \mathrm{N}-8^{\circ} 21^{\prime} 55^{\prime \prime}$ W. A : $105 \mathrm{~m}$; P : 44,5\%; $1: 1$ à $1,5 \mathrm{~m} ;$ pr.: 20 à $40 \mathrm{~cm}$. Ruisseau assez rapide; sable, vase; granite; cultures, avec arbres sur les deux rives ; $15,5^{\circ} \mathrm{C}$ le 4.10 .1972 .

Station 7. - Affluent de la Ribeira de Setores, à Areias de Vilar.

$41^{\circ} 32^{\prime} 18^{\prime \prime} \mathrm{N}-8^{\circ} 33^{\prime} 10^{\prime \prime} \mathrm{W}$. A : $35 \mathrm{~m} ; \mathrm{P}: 20 \%$; 1: $0,5 \mathrm{~m} ;$ pr: 50 à $70 \mathrm{~cm}$. Rigole en terre, courant modéré ; sable avec végétation sur les bords; schistes et granite ; cultures d'un côté, haut mur de l'autre; $12^{\circ} \mathrm{C}$ le 11.12.1968.

Station 8. - Rio Ave, à Serra da Cabreira.

$41^{\circ} 36^{\prime} 28^{\prime \prime} \mathrm{N}-8^{\circ} 02^{\prime} 17^{\prime \prime}$ W. A : $770 \mathrm{~m} ; \mathrm{P}: 50 \% ; \mathrm{l}: 1$ à $1,5 \mathrm{~m} ; \mathrm{pr}: 20$ à $40 \mathrm{~cm}$. Ruisseau de montagne, dans la région des sources de la rivière Ave, courant assez rapide; graviers, sable et vase, avec quelques Renonculacées; granite; brousse sur les deux rives; $18,5^{\circ} \mathrm{C}$ le 4.7.1968.

Station 9. - Ribeira de Pereiros, à Póvoa de Lanhoso.

$41^{\circ} 34^{\prime} 16^{\prime \prime} \mathrm{N}-8^{\circ} 15^{\prime} 40^{\prime \prime} \mathrm{W}$. A : $170 \mathrm{~m} ; \mathrm{P}: 11,5 \%$; $1: 0,8$ à $1,5 \mathrm{~m} ; \mathrm{pr}: 20$ à $40 \mathrm{~cm}$. Ruisseau lent ; sable, vase; granite; cultures sur les deux rives ; prélèvement le 23:10.1968.

Station 10. - Ribeira de Costas-Antas, à Gontim.

$41^{\circ} 31^{\prime} 26^{\prime \prime} \mathrm{N}-8^{\circ} 5^{\prime} 59^{\prime \prime}$ W. A : $650 \mathrm{~m} ; \mathrm{P}: 32 \% ; \mathrm{l}: 1$ à $1,5 \mathrm{~m} ; \mathrm{pr}: 20$ à $40 \mathrm{~cm}$. Ruisseau à vitesse modérée; fond rocheux avec du sable, végétation aquatique avec des mousses et Oenanthe crocata L.; granite; prairie sur une rive, bois de pins sur l'autre; $12,5^{\circ} \mathrm{C}$ le 9.10 .1972 .

Station 11. - Ribeira de Costas-Antas, à Felgueiras.

$41^{\circ} 30^{\prime} 58^{\prime \prime} \mathrm{N}-8^{\circ} 6^{\prime} 35^{\prime \prime} \mathrm{W}$. A : $600 \mathrm{~m} ; \mathrm{P}: 20 \% 0 ; 1: 1$ à $1,5 \mathrm{~m} ; \mathrm{pr}: 20$ à $40 \mathrm{~cm}$. Ruisseau à courant modéré ; graviers, sable, avec quelques mousses; granite ; bois de pins et eucalyptus sur les deux rives; $12,5^{\circ} \mathrm{C}$ le 9.10 .1972 . 
Station 12. - Rio Vizela, à Golâes.

$41^{\circ} 27^{\prime} 36^{\prime \prime} \mathrm{N}-8^{\circ} 11^{\prime} 1^{\prime \prime}$ W. A : $250 \mathrm{~m} ; \mathrm{P}: 2,2 \%$; $1: 4$ à $5 \mathrm{~m}$; pr: 40 à $70 \mathrm{~cm}$. Petite rivière à courant modéré ; galets, graviers et sable, végétation aquatique parfois abondante (Renonculacées et Oenanthe); granite; cultures avec murs de soutien sur les deux rives; $17,5^{\circ} \mathrm{C}$ le $1.8 .1968,14{ }^{\circ} \mathrm{C}$ le $17.9 .1968,19^{\circ} \mathrm{C}$ le 28.8 .1969 . Station 13. - Rio Beça, à Serraquinhos.

$41^{\circ} 47^{\prime} 2^{\prime \prime} \mathrm{N}-7^{\circ} 39^{\prime} 17^{\prime \prime} \mathrm{W}$. A : $880 \mathrm{~m} ; \mathrm{P}: 5 \% ; 1: 3$ à $4 \mathrm{~m} ;$ pr: 30 à $50 \mathrm{~cm}$. Ruisseau lent; sable, vase, végétation aquatique (Renonculacées surtout); granite; prairies bordées d'arbres sur les deux rives; $15,5^{\circ} \mathrm{C}$ le 15.6.1973.

Station 14. - Rio Beça, à Barracâo.

$41^{\circ} 45^{\prime} 43^{\prime \prime} \mathrm{N}-7^{\circ} 42^{\prime} 8^{\prime \prime} \mathrm{W} . \mathrm{A}: 810 \mathrm{~m}$; P: $5 \% ; 1: 1$ à $2 \mathrm{~m} ;$ pr. $: 30$ à $50 \mathrm{~cm}$. Ruisseau à courant modéré, mais présence de rapides en amont ; graviers, sable, avec des mousses; schistes métamorphiques; prairie bordée d'arbres sur une rive, bois sur l'autre; $20^{\circ} \mathrm{C}$ le 13.7 .1972 et $8,5^{\circ} \mathrm{C}$ le 18.4.1973.

Station 15. - Rio Olo, à Ermelo.

$41^{\circ} 21^{\prime} 51^{\prime \prime} \mathrm{N}-7^{\circ} 53^{\prime} 32^{\prime \prime}$ W. A : $290 \mathrm{~m} ; \mathrm{P}: 10,5 \% ; 1: 7$ à $8 \mathrm{~m} ;$ pr : 50 à $80 \mathrm{~cm}$. Rivière de montagne à courant rapide; gros galets, quelques mousses; schistes et granite; rives très inclinées, avec brousse ; $18,5^{\circ}{ }^{\circ} \mathrm{C}$ le 31.7:1973.

Station 16. - Ribeira de Lila, à Eixes.

$41^{\circ} 32^{\prime} 20^{\prime \prime} \mathrm{N}-7^{\circ} 15^{\prime} 40^{\prime \prime}$ W. A : $280 \mathrm{~m} ; \mathrm{P}: 3,5^{\prime \prime}$; $; 1: 3$ à $5 \mathrm{~m} ;$ pr : 30 à $50 \mathrm{~cm}$. Ruisseau lent; petits galets, graviers, sable, végétation aquatique (Oenanthe surtout); schistes et granite ; terres de culture ; $21^{\circ} \mathrm{C}$ le 29.5 .1973 .

Station 17. - Rio Caima, à Albergaria das Cabras.

$40^{\circ} 51^{\prime} 46^{\prime \prime} \mathrm{N}-8^{\circ} 17^{\prime} 43^{\prime \prime}$ W. A : $900 \mathrm{~m} ; \mathrm{P}: 40^{\prime \prime} ; 1: 3$ à $4 \mathrm{~m} ;$ pr: 30 à $50 \mathrm{~cm}$. Petite rivière à courant modéré; galets, sable et rochers; granite; prairie sur les deux rives; prélèvement le 12.8.1968.

Station 18. - Rio Zezere, à Fonte Santa.

$40^{\circ} 22^{\prime} 51^{\prime \prime} \mathrm{N}-7^{\circ} 32^{\prime} 46^{\prime \prime} \mathrm{W}$. A : $880 \mathrm{~m} ; \mathrm{P}: 80 \% ; 1: 5$ à $7 \mathrm{~m} ;$ pr : 40 à $80 \mathrm{~cm}$. Rivière de montagne à courant rapide; gros galets et rochers; granite ; prélèvement le 24.7.1973.

Station 19. - Ribeira de Nisa, à Portalegre.

$39^{\circ} 19^{\prime} 50^{\prime \prime} \mathrm{N}-7^{\circ} 25^{\prime} 40^{\prime \prime}$ W. A : $470 \mathrm{~m} ; 1: 1,5$ à $2 \mathrm{~m}$; pr. 30 à $40 \mathrm{~cm}$. Ruisseau lent ; graviers, sable et vase ; granite ; prélèvement le 20.5:1972.

Station 20. - Ribeira de Odelouca, à Sâo Marcos da Serra.

$37^{\circ} 21^{\prime} 30^{\prime \prime} \mathrm{N}-8^{\circ} 22^{\prime} 30^{\prime \prime}$ W. A : $100 \mathrm{~m}$; P: $4 \%$ \% ; 1: 5 à $7 \mathrm{~m} ;$ pr: 20 à $30 \mathrm{~cm}$. Ruisseau très lent; graviers et vase argileuse, avec des algues; terrains argileux carbonifères ; $19^{\circ} \mathrm{C}$ le 18.5 .1972 .

Les Haenydra, les Hydraena s. s. et les Elmidae sont tous bien représentés dans le peuplement équilibré de la Ribeira de Costas Antas à 650 et $600 \mathrm{~m}$ (stations 10 et 11). La Ribeira de Casaldelo (station 6), située à plus basse altitude mais à pente encore plus forte, est plus riche en Elmis. A l'opposé, le Rio Beça à 880 et $810 \mathrm{~m}$ (stations 13 et 14 ) présente une pente plus faible. Limnius perrisi mediocarinatus n'y a pas été récolté mais on voit apparaître des espèces comme Normandia nitens, Oulimnius rivularis et même Stenelmis canaliculata qui tolèrent un plus fort réchauffement estival. Le prélèvement assez incomplet de la station 1 peut être rattaché aux précédents.

Le Rio Castro-Laboreiro à $935 \mathrm{~m}$ (station 2) constitue une transition entre les ruisseaux à forte pente déjà cités (stations 10,11 et 6 ) et les petites rivières (stations $3,5,12,15$ et 18). Ces dernières ont un peuplement voisin de celui du Rio Beça (stations 13 et 14), mais un peu moins riche, semble-t-il, en Hydraenidae. 


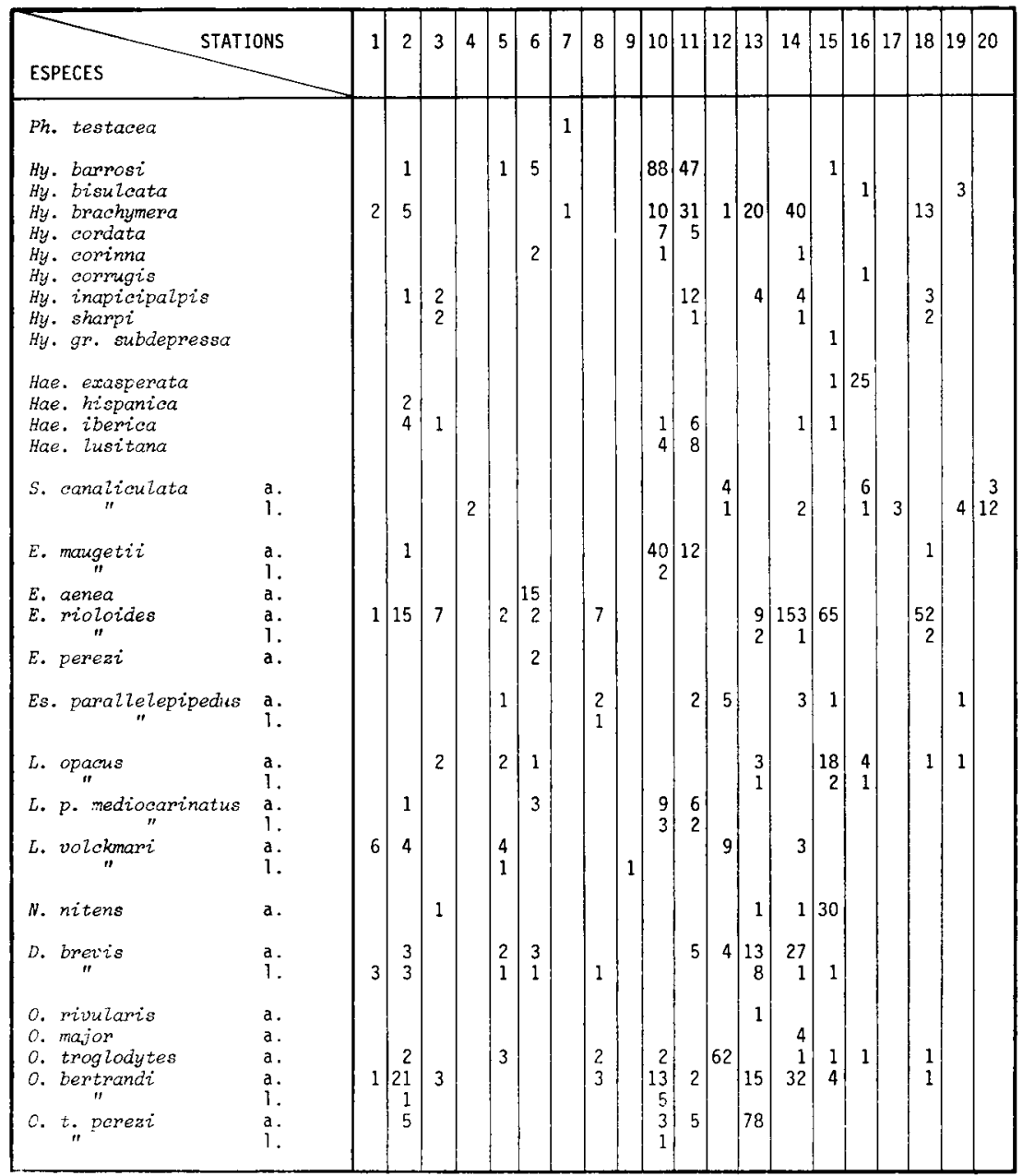

TableaU I. - Répartition des Hydraenidae et Elmidae dans les stations prospectées. a.: adultes; l. : larves. D. : Dupophilus ; E : Elmis ; Es.: Esolus ; Hae. : Haenydra; Hy.: Hydraena s. s.; L.: Limnius ; N.: Normandia ; O : Oulimnius; Ph. : Phothydraena; S. : Stenelmis.

Enfin, le groupement de la station 16 est intermédiaire entre celui du Rio Beça (stations 13 et 14) et ceux des stations 4, 7, 9, 19 et 20. Ces cours d'eau de basse altitude ou à courant lent renferment surtout des Stenelmis, ainsi que Phothydraena testacea. Ils correspondent à un milieu différent, partiellement prospecté.

L'ensemble donne une image assez complète du peuplement des ruisseaux et petites rivières qui coulent à moyenne altitude sur des roches en majorité cristallines. D'après les récoltes d'A. d'Orchymont et d'H. Bertrand, ces cours d'eau renferment sans doute aussi des 
Esolus angustatus (Muiller, 1821), dans les zones amont, ainsi que des Hydraena muscicoles comme $H$. minutissima Stephens, 1829, H. pygmaea Waterhouse, 1833 et $H$. stussineri Kuwert, 1888. Cette dernière remplace $H$. angulosa Mulsant, 1844 dans la Péninsule Ibérique. En aval, on peut s'attendre à rencontrer des Limnius intermedius Fairmaire, 1881, des Esolus pygmaeus (Müller, 1806) et une plus grande abondance d'Oulimnius rivularis et de Stenelmis.

\section{2. - HYDRAENIDAE}

L'un de nous (Berthélemy 1965) avait remarqué que les sous-genres Haenydra et Phothydraena seraient sans doute élevés plus ou moins rapidement au rang de genres. Cette mesure a été prise par Ienistea (1968) et, au moins pour les Haenydra, approuvée par Janssens (1967 a, b, 1972). Janssens, d'autre part, a créé en 1972 le genre Hydraenopsis pour des espèces orientales et éthiopiennes. Hydraena quadricollis Wollaston, 1864 (= Hydraena nilotica Rey, 1886 - voir d'Orchymont 1940) doit être rangée dans ce nouveau genre. Hydraenopsis atteint donc la frange méridionale de la région paléarctique.

Les genres Haenydra, Phothydraena et Hydraenopsis sont basés sur des caractères évolués communs (synapomorphes, Hennig 1966). Ils sont bien monophylétiques, selon l'acception d'Hennig ( $=$ holophylétiques, Ashlock 1971, 1974). Mais le genre Hydraena s. s., ainsi restreint, n'est défini par rapport à Haenydra, Phothydraena et Hydraenopsis que par des caractères primitifs (symplésiomorphes). C'est un genre paraphylétique et il conviendra de tenir compte de ce fait dans toute étude biogéographique.

\subsection{PHOTHYDRAENA}

La seule Phothydraena rencontrée ici, Ph. testacea (Curtis, 1830), se rencontre de l'Afrique du Nord aux Iles Britanniques et à l'Europe orientale. Elle paraît répandue dans toute la Péninsule Ibérique (d'Orchymont $1936 \mathrm{~b}$ ). Les déterminations anciennes, toutefois, devront être vérifiées grâce à un examen des édéages. Comme en France, cette espèce a pu être confondue avec $P h$. atrata (Desbrochers des Loges, $1891)^{3}$.

\subsection{HYDRAENA S. S.}

Toutes les Hydraena récoltées sont des espèces ou sous-espèces ibériques.

3. Rappelons que Ph. paganettii (Ganglbauer, 1901) d'Europe centrale est une espèce distincte de $P h$. atrata (voir Binaghi 1965). 
$H$. brachymera d'Orchymont, 1936 remplace une espèce voisine très répandue dans le reste de l'Europe, $H$. riparia Kugelann, 1792. Toutes deux ont été capturées dans un même ruisseau du Pays Basque français. Cette coexistence confirme leur isolement spécifique proposé par l'un de nous (Berthélemy 1965). H. brachymera présente les mêmes caractères écologiques qu' $H$. riparia. Il s'agit d'un cas typique de vicariance géographique.

Il en est de même d'H. cordata Schaufuss, 1883, de la Péninsule Ibérique, et d'H. cordata regularis Rey, 1885, de France, ainsi que du couple $H$. stussineri Kuwert, $1888-H$. angulosa Mulsant, 1844. $H$. stussineri ne figure pas dans nos récoltes mais existe certainement dans le territoire étudié.

Par contre, les autres Hydraena capturées ne possèdent pas de proches parents au nord des Pyrénées. Ces Hydraena sont $H$. corinna d'Orchymont, 1936, $H$. corrugis d'Orchymont, 1935, H. inapicipalpis Pic, 1918, H. barrosi d'Orchymont, 1935, $H$. bisulcata Rey, 1884 et $H$. sharpi Rey, 1880.

$H$. sharpi, en particulier, ne présente pas d'affinités réelles avec H. pulchella Germar, 1824, contrairement à l'opinion d'A. d'Orchymont (1936 b). Le «Phylum pulchella» d'A. d'Orchymont (1944) n'est défini que par le nombre réduit des stries élytrales. Il a surtout une valeur pratique (Janssens 1965) et semble polyphylétique. Pour obtenir un groupe monophylétique, il est nécessaire d'utiliser un caractère moins sujet à convergence, tel que la chétotaxie de l'édéage. Un "groupe pulchella », plus restreint et plus homogène, peut être basé sur la présence de plus de quatre soies à l'apex de la partie proximale. Ce "groupe pulchella" renferme, outre $H$. pulchella, H. phassilyi d'Orchymont, 1931, H. çanakçioglui Janssens, 1965, ainsi que $H$. phallerata d'Orchymont, 1944, $H$. philyra d'Orchymont, 1944 et $H$. byzantina Janssens, 1965. Les trois dernières espèces forment un sous-groupe dont la partie proximale de l'édéage porte une touffe de soies coudées insérées vers le milieu de l'organe.

Chez H. sharpi, au contraire, la partie proximale de l'édéage ne présente que quatre soies, ce qui constitue l'ornementation primitive (Eerthélemy 1965) La partie distale est plus longue que dans le "groupe pulchella " et les soies des paramères n'ont pas la même distribution. Les affinités véritables d'H. sharpi devront être précisées ultérieurement. Jusqu'alors, $H$. sharpi n'avait été capturée que dans la Sierra de Guadarrama.

\subsection{HAENYDRA}

La découverte d'une Haenydra nouvelle, $H$. lusitana, porte à six le nombre des espèces de ce genre capturées dans la Péninsule Ibérique. Sans doute peut-on ajouter à ces six espèces $H$. truncata (Rey, 1885) qui vit certainement dans les Pyrénées espagnoles. 
Les Haenydra ne représentent que le quart du total des espèces de Phothydraena, d'Hydraena s. s. et d'Haenydra. En Europe centrale, elles constituent plus du tiers de cet ensemble. Les Haenydra se raréfient de même dans le Sud de la Péninsule Balkanique (d'Orchymont 1943). Janssens (1965) attribue ce fait à la prédominance des terrains calcaires en Grèce.

L'hypothèse de Janssens ne s'applique pas à l'Espagne et au Portugal. En réalité, une nette préférence pour les terrains siliceux n'a été démontrée jusqu'ici que pour une seule espèce d'Haenydra, $H$. dentipes (Ahrens et Germar, 1844) par d'Orchymont (1938). La plus commune des Haenydra, $H$. gracilis, est abondante dans certaines régions calcaires. Une autre Haenydra, $H$. polita (Kiesenwetter, 1849) semble même plutôt calcicole (Berthélemy 1966). Il est possible que d'autres espèces d'Haenydra soient sensibles à la nature des terrains mais il n'existe pas, dans ce domaine, de caractère générique.

Les Haenydra se rencontrent surtout dans les régions accidentées et boisées, en amont des zones habitées par les Hydraena s. s. Cette répartition écologique suffit à expliquer leur moindre abondance dans le Sud de l'Europe.

Nous présentons ci-dessous une mise au point sur toutes les Haenydra ibériques, qu'elles soient ou non présentes dans nos récoltes. Les caractères choisis pour définir le groupe gracilis et ses sousgroupes sont, nous l'espérons, synapomorphes.

\subsubsection{Groupe gracilis}

- Soie droite de la partie proximale de l'édéage réduite ou nulle.

- Partie distale comprenant :

a) une lame appliquée contre la partie proximale (fig. 2 à 8,1 .),

b) une pièce intermédiaire articulée avec la précédente (fig. 2 à 8 , i),

c) un élément récurrent fixé à la pièce intermédiaire (fig. 2 à 8, r.).

2.3.1.1. H. gracilis (Germar, 1824).

- Élément récurrent court, avec deux lobes verticaux au-delà de l'orifice du canal déférent (Berthélemy 1964 a, fig. 9).

D'après d'Orchymont (1936 b), la présence d' $H$. gracilis dans la Péninsule Ibérique demandait confirmation. $H$. gracilis a récemment été capturée par le $\mathrm{D}^{\mathrm{r}}$ Puthz dans la Province de Santander. H. gracilis est présente dans tout le reste de l'Europe, à l'exception de la Péninsule Italienne au-delà du Frioule (Binaghi 1963). Sa limite méridionale dans la Péninsule Ibérique devra être précisée.

2.3.1.2. H. emarginata (Rey, 1885) - H. saga (d'Orchymont, 1930) - H. samnitica (Friori, 1904) - H. sp. (= emarginata sensu Capellaro, 1959 nec Rey). 


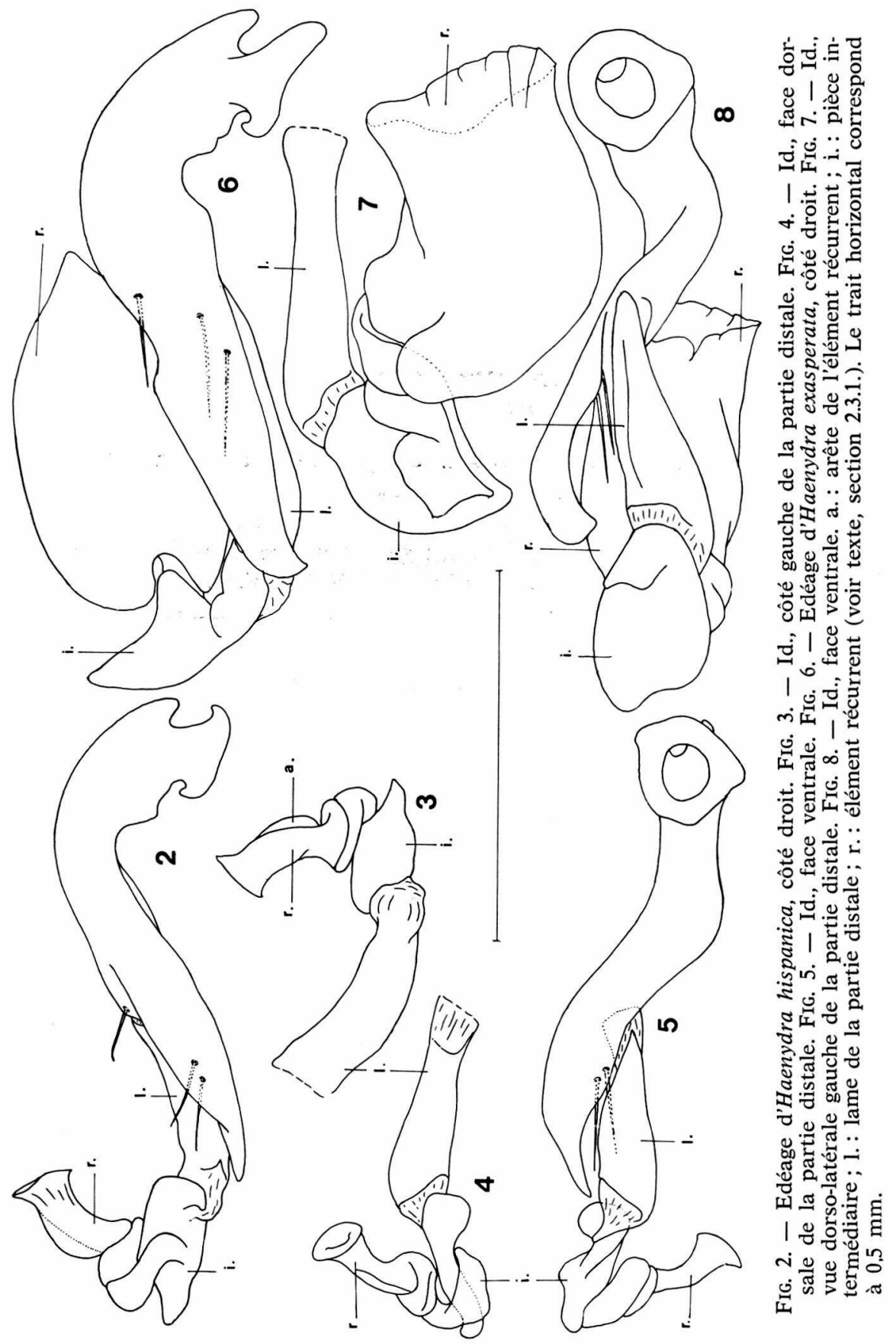


- Partie proximale tronquée à l'extrémité (Berthélemy, 1964 a, fig. 1, 7 et '14).

Ce sous-groupe est représenté dans les Monts Cantabriques par $H$. emarginata. Rappelons qu' $H$. emarginata et $H$. saga sont des espèces distinctes, et non des races comme l'indique Lohse (Freude, Harde et Lohse 1971). Le problème des races italiennes et balkaniques d' $H$. saga - ou des espèces rassemblées sous ce nom - n'est pas encore résolu (Pretner 1970). Mais $H$. emarginata et $H$. saga cohabitent dans les mêmes cours d'eau sans se mélanger et leur distinction spécifique ne peut être remise en cause.

2.3.1.3. H. hispanica (Ganglbauer, 1901) - H. belgica (d'Orchymont, 1930) - H. dalmatina (Ganglbauer, 1901).

- Tube de l'élément récurrent pourvu d'une arête dorsale (fig. 2 à 5 , a.).

H. hispanica n'avait été capturée jusqu'alors que sur le versant nord de la Sierra de Guarrama. Sa présence dans la station 2 confirme son caractère alticole.

$H$. belgica est très proche d'H. hispanica. Elle s'étend du Massif Central à la Bulgarie. $H$. dalmatina se rencontre dans le Sud de la Dalmatie, au Monténégro et dans le Nord de la Grèce.

La coexistence d'H. belgica et d'H. dalmatina dans le Sud de la Bosnie ne peut être exclue. D'après les données actuelles, toutefois, les trois espèces sont strictement allopatriques.

2.3.1.4. H. exasperata (d'Orchymont, 1936) - H. excisa (Kiesenwetter, 1849) - H. phallica (d'Orchymont, 1930) - H. cata (d'Orchymont, 1943) - H. caucasica (Kuwert, 1888).

- Partie récurrente de fort diamètre (fig. 6 à 8 ).

$H$. exasperata vit en Espagne et au Portugal, $H$. excisa en Europe moyenne et orientale, $H$. phallica en Serbie, Bulgarie et Roumanie, $H$. cata à Lesbos et près de la mer de Marmara, H. caucasica au Caucase.

II s'agit donc de formes en grande partie allopatriques, avec, cependant, coexistence d'H. excisa et d'H. phallica en Europe orientale (Ienistea 1968).

Les Haenydra du groupe gracilis constituent un exemple assez schématique de spéciation géographique.

$H$. gracilis est l'espèce la plus tolérante. Elle descend assez bas vers l'aval des cours d'eau. Son aire de répartition est continue et ses caractères morphologiques homogènes.

Les autres espèces sont plus localisées. Les formes souches de chacun des trois sous-groupes se sont répandues d'un massif à l'autre à la faveur de refroidissements climatiques. Les réchauffements ultérieurs ont isolé en altitude des populations et interrompu les échan- 
ges génétiques. Il en est résulté une divergence plus ou moins poussée des caractères morphologiques.

\subsubsection{Haenydra iberica}

Comme nous l'avions supposé (Berthélemy 1966), H. iberica est voisine d'H. truncata.

Chez les deux espèces, la partie distale de l'édéage se termine par un tube allongé, le rebord élytral est étroit, l'extrémité des élytres légèrement tronquée chez le mâle, non échancrée chez la femelle, les tibias postérieurs du mâle portent une frange très fournie de longues soies.

Le groupe d'H. truncata renferme aussi une espèce balkanique, $H$. vedrasi (d'Orchymont, 1931). Le mâle d'H. vedrasi possède des tibias antérieurs élargis et incurvés. Ce caractère sexuel secondaire n'est mentionné par aucun des auteurs qui ont vu cette espèce (d'Orchymont 1931, Derenne 1954, Janssens 1965, Pretner 1970 sub nom. $H$. montenegrina syn. nov.).

$H$. iberica, $H$. truncata et $H$. vedrasi forment un ensemble d'espèces allopatriques. Les différences entre ces trois espèces sont plus prononcées qu'entre les membres d'un même sous-groupe du groupe gracilis. Leur divergence semble plus ancienne.

\subsubsection{Haenydra lusitana nov. sp. ${ }^{4}$}

Haenydra de petite taille. Chez les deux sexes, pronotum de 0,36 à $0,43 \mathrm{~mm}$ de long et 0,49 à $0.54 \mathrm{~mm}$ de large ; élytres de 1,09 à $1,15 \mathrm{~mm}$ de long et 0,63 à $0,68 \mathrm{~mm}$ de large, pris ensemble.

Face dorsale unicolore, brun-jaune, brillante entre les points, sauf le clypeus qui est finement chagriné.

Bombement des élytres, striation et rebord explané comme chez H. gracilis.

Mâle : fémurs dilatés, tibias intermédiaires denticulés, sans échancrure (fig. 19); tibias postérieurs avec une frange de longues soies clairsemées.

Au-delà de l'insertion de la partie distale, la partie proximale de l'édéage est aplatie en lame un peu concave vers la gauche et vers le dos. Les quatre soies, bien développées, s'insèrent à peu près au même niveau, le long de l'organe (fig. 13).

La partie distale, peu sclérifiée, dépasse la partie proximale. Le canal déférent décrit une demi-boucle et s'ouvre vers la droite (fig. 16).

4. Description C. Berthélemy. 

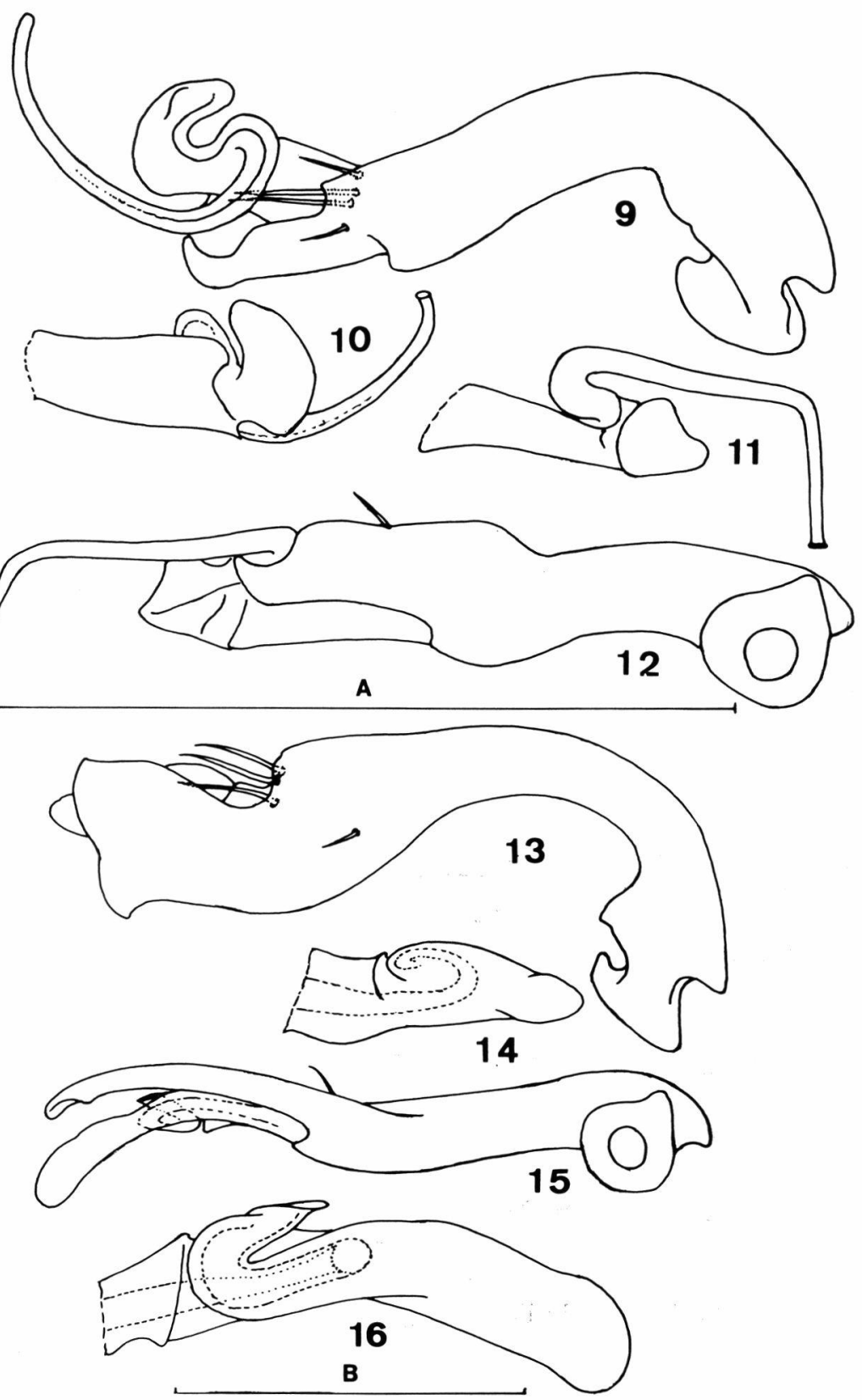

Fig. 9. - Edéage d'Haenydra iberica, côté droit. Fig. 10. - Id., côté gauche de la partie distale. Fig. 11. - Id., face dorsale de la partie distale. FIG. 12. Id., face ventrale. FIG. 13. - Edéage d'Haenydra lusitana nov. sp., côté droit. FIg. 14. - Id., côté gauche de la partie distale. Fig. 15. - Id., face ventrale. Fig. 16. - Id., face dorsale de la partie distale. Le trait A correspond à $0,5 \mathrm{~mm}$ (fig. 9 à 15), le trait $\mathrm{B}$ à $0,1 \mathrm{~mm}$ (fig. 16). 
Cette structure de l'édéage, notamment de la partie distale, permet de distinguer aisément $H$. lusitana de toutes les autres Haenydra.

Femelle. Nous nous bornerons à comparer $H$. lusitana aux femelles des autres Haenydra ibériques.

L'échancrure des élytres est plus obtuse que chez $H$. gracilis, $H$. saga, $H$. emarginata et $H$. exasperata. Le rebord élytral est plus étroit que chez les trois dernières espèces citées. A l'opposé, ce rebord est plus large que chez $H$. iberica et $H$. truncata. Chez ces deux dernières espèces, les élytres ne sont pas échancrés. Enfin, les points des élytres sont moins profonds chez $H$. lusitana que chez $H$. hispanica et le tégument moins chagriné, surtout sur le clypeus.
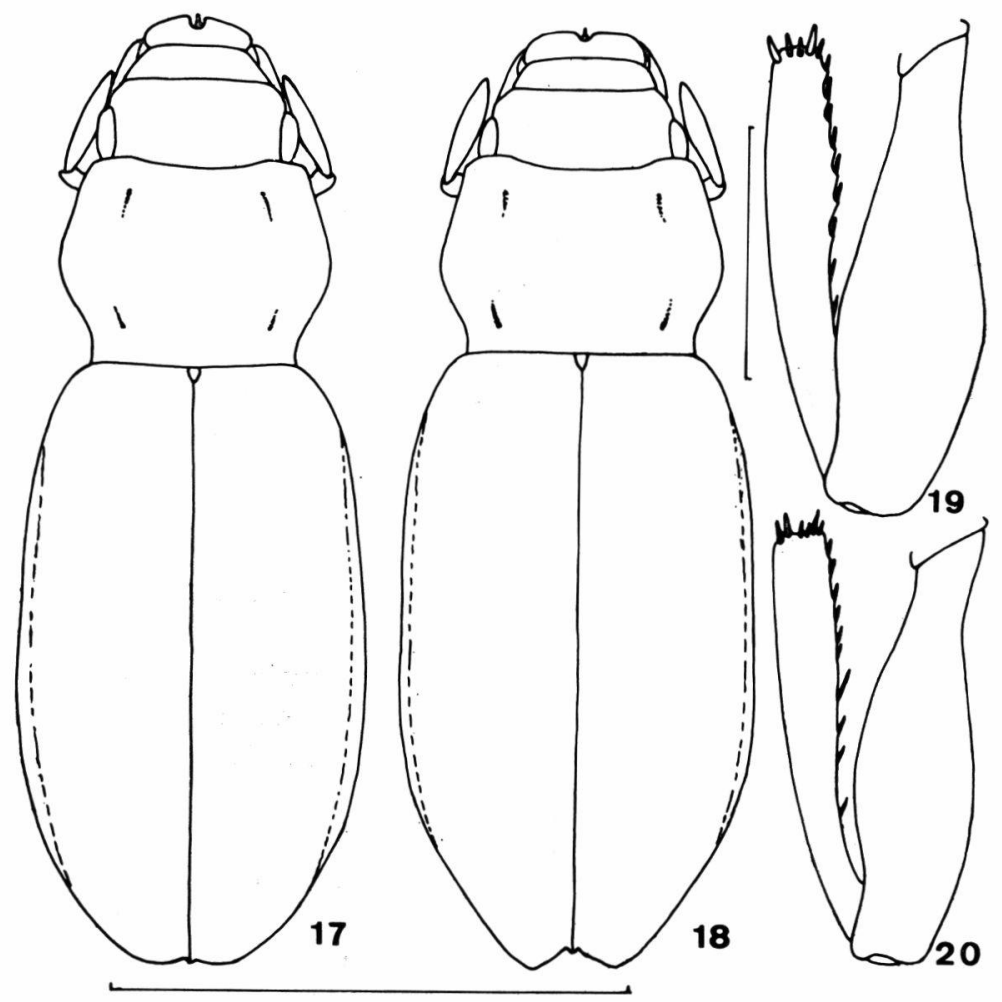

Fig. 17 à 20. - Haenydra lusitana nov. sp. Fig. 17. - Face dorsale, mâle. Fig. 18. - Id., femelle. Fig. 19. - Fémur et tibia mésothoraciques gauches, mâle. Fig. 20. - Id., femelle. Le trait horizontal correspond à $1 \mathrm{~mm}$ ( $f$ ig. 17 et 18 ), le trait vertical à $0,2 \mathrm{~mm}$ ( $f$ ig. 19 et 20 ).

Les dimensions des Haenydra ne présentent que de faibles variations intraspécifiques. Aussi la petite taille d'H. lusitana permet-elle de la distinguer aisément des autres Haenydra ibériques. A cet égard, ainsi que par sa coloration, H. lusitana rappelle des Haenydra étran- 
gères à la péninsule, telles qu'H. schuleri (Ganglbauer, 1901), H. scitula (d'Orchymont, 1947) et $H$. carniolica (Pretner, 1970). H. lusitana est plus convexe que les trois espèces citées. La structure des édéages montre qu'elle ne présente d'affinités réelles avec aucune d'entre elles.

Matériel étudié : un holotype mâle capturé dans la Ribeira de Costas-Antas à Felgueiras, sept paratypes de même provenance et quatre paratypes récoltés dans la Ribeira de Costas-Antas à Gontim (collection C. Berthélemy).

\section{3. - ELMIDAE}

Stenelmis canaliculata (Gyllenhål, 1808) tolère mieux les faibles courants que la plupart des autres Elmidae. On la rencontre aussi bien dans la zone de ressac des lacs que dans les grands cours d'eau ou, comme ici, dans les ruisseaux à faible pente.

Elmis maugetii Latreille, 1798 se trouve dans toute la Péninsule Ibérique. Chez les exemplaires du Portugal, les interstries impaires sont moyennement surélevées et non fortement carénées comme chez ceux de la Sierra de Guadarrama (forme "similis " Flach, 1882).

Elmis aenea (Müller, 1806) n'a pas encore été identifiée avec certitude au sud du Douro. Mais il ne s'agit sans doute pas de sa véri. table limite méridionale.

Eimis rioloides (Kuwert, 1890) avait été signalée du Portugal par Kuwert (1890) sous le nom de Lareynia fossulata et par Bertrand (1960) sous celui d'Elmis coiffaiti. Vers le sud, cette espèce atteint Algeciras. C'est la plus abondante des Elmis récoltées ici. Cette prédominance confirme son affinité pour les terrains cristallins (Berthélemy 1966, sub nom. E. coiffaiti).

Elmis perezi Heyden, 1870, enfin, a été retrouvée près de sa localité typique, la Serra do Gerez. Elle est fréquente dans les ruisselets et ruisseaux à forte pente du Pays Basque. Vers l'est, elle atteint la vallée d'Ossau.

L'ornementation de la larve de Limnius perrisi mediocarinatus Berthélemy, 1964 est voisine de celle de L. perrisi perrisi Dufour, 1843. Elle confirme le rang subspécifique attribué à cette forme. Cette sous-espèce vit dans les Monts Ibériques, le Nord du Portugal et la partie occidentale des Monts Cantabriques. C'est la plus répandue des trois races ibériques de L. perrisi. L. perrisi carinatus (PerezArcas, 1866) est propre à la Sierra de Guadarrama tandis que $L$. perrisi subcarinatus (Sharp, 1872) n'a été nencontrée que près des sources de l'Ebre. Cette dernière sous-espèce est une forme de passage entre $L$. perrisi mediocarinatus et les $L$. perrisi perrisi des Pyrénées.

La transition entre Oulimnius tuberculatus perezi (Sharp, 1872) et O. tuberculatus tuberculatus (Müller, 1806) s'effectue peut-être dans la même région. En effet, alors que l'on trouve encore O. tuberculatus 
perezi au nord de León, une femelle récoltée près d'Alsasua ne diffère des $O$. tuberculatus tuberculatus des Pyrénées que par un bombement plus marqué. Ce point devra être confirmé par l'étude de mâles des mêmes régions.

Toutes les localités d'Oulimnius major (Rey, 1889) publiées précédemment étaient situées en France (Berthélemy 1962, 1964 b, c). $O$. major vit aussi au Portugal et en Andalousie, où il avait été récolté par A. d'Orchymont. Ces données nouvelles étendent son aire de répartition de plus de mille kilomètres.

Les habitats des diverses espèces d'Oulimnius sont peu distincts les uns des autres. L'endémique ibérique $O$. bertrandi Berthélemy, 1964 ne fait pas exception à la règle. Il cohabite avec $O$. rivularis (Rosenhauer, 1856), O. major, O. troglodytes (Gyllenhål, 1827) et $O$. tuberculatus perezi. Il semble toutefois plus alticole que les deux premiers.

Dupophilus brevis Mulsant et Rey, 1872 est, comme en France, associé à Elmis rioloides en terrain siliceux.

Les préférences éventuelles de Normandia nitens (Müller, 1817) devront être précisées. Cette espèce était rangée naguère dans le genre Riolus. Mais elle n'est peut-être pas aussi étroitement liée aux terrains calcaires que les véritables Riolus. Les $N$. nitens récoltées ici sont brachyptères et leurs élytres sont dépourvus de bosse humérale.

'Enfin, comme Elmis maugetii, Esolus parallelepipedus (Müller, 1806), Limnius opacus Müller, 1806, L. volckmari (Panzer, 1793), Oulimnius rivularis et $O$. troglodytes atteignent le Sud de la Péninsule Ibérique. Au point de vue écologique comme au point de vue biogéographique, leur présence dans les cours d'eau étudiés est en accord avec les données antérieures.

\section{4. - CONCLUSION}

Les peuplements d'Elmidae décrits ci-dessus correspondent bien à ceux que l'on rencontre dans les cours d'eau comparables des Pyrénées ou du Massif Central français. La seule particularité est la présence d'abondants Oulimnius bertrandi qui n'ont pas de contrepartie au nord de l'Ebre.

Les peuplements d'Hydraenidae, par contre, sont presque exclusivement constitués d'endémiques, dont quelques-uns atteignent tout au plus le Pays Basque. Cette différence entre les deux groupes de Coléoptères torrenticoles a déjà été signalée par l'un de nous (Berthélemy 1966, 1969). Elle souligne le caractère complémentaire des deux groupes pour des études hydrobiologiques: les Elmidae facilitent les comparaisons écologiques, alors que les Hydraenidae montrent quel est le degré d'isolement d'une région géographique. 


\section{TRAVAUX CITÉS}

Ashlock (P. D.). 1971. - Monophyly and associated terms. Syst. Zool., 16: 63-69. Ashlock (P. D.). 1974. - The uses of cladistics. A. Rev. Ecol. Syst., 5: 81-99.

BERTHÉlemy (C.). 1962. - Contribution à l'étude systématique des Elminthidae (Coléoptères). Bull. Soc. Hist. nat. Toulouse, 97 : 201-225.

BERTHÉlEMY (C.). 1964 a. - Sur la position systématique de quelques Hydraena européennes (Coléoptères). Bull. Soc. Hist. nat. Toulouse, 99 : 175-185.

BerthÉlemy (C.). 1964 b. - Elminthidae d'Europe occidentale et d'Afrique du Nord (Coléoptères). Bull. Soc. Hist. nat. Toulouse, 99 : 244-285.

Berthélemy (C.). 1964 c. - Les Elminthidae décrits par C. Rey: Note synonymique (Coléoptères Dryopoidea). Bull. Soc. Hist. nat. Toulouse, 99 : 525-528.

BERTHÉlemy (C.). 1965. - Note taxonomique et faunistique sur des Hydraena françaises et ibériques (Coléoptères). Annls Limnol., 1 : 3:19.

BERTHÉLEMY (C.). 1966. - Recherches écologiques et biogéographiques sur les Plécoptères et Coléoptères d'eau courante (Hydraena et Elminthidae) des Pyrénées. Annls Limnol., 2 : 227-458.

BERTHÉLEMY (C.). 1969. - Sur quelques Hydraena et Elminthidae de Grèce (Coléoptères). Biologia Gallo-Hellenica, 2 : 35-43.

BERTRAND (H.). 1965. - Récoltes de Coléoptères aquatiques dans les régions montagneuses de l'Espagne: observations écologiques (Dryopidae, Elminthinae = Helminae Auct.). Annls Limnol., 1 : 245-255.

Binaghi (G.). 1963. - Materiali per lo studio delle Hydraena italiane. Le $H y$. draena del Friuli (Studio ecologico, biogeografico e statistico) $\left(5^{e}\right.$ contributo). Memorie Mus. civ. Stor. nat. Verona, $11: 17-48$.

BinaGH (G.). 1965. - Materiali per lo studio delle Hydraena italiane ( $6^{\mathrm{c}}$ contributo). Memorie Soc. ent. ital., $44: 12-22$.

Capellaro (G.). 1959. - Riperti di Hydraena in Piemonte (Coleopt. Hydrophilidae). Boll. Soc. ent. ital., 89 : 54-57.

DERENNE (E.). 1954. - Hydraenidae et Hydrophilidae recueillis par M. Emile Janssens au Mont Pélion (Grèce). Bull. Inst. r. Sci. nat. Belg., 30 (20) : 1-4.

FlaCh (C.). 1882. - Variabilität der Lareynia-Arten. Dt. ent. Z., 26: 249-253.

Freude (H.), Harde (K. W.) et Lohse (G. A.). 1971. - Die Käfer Mitteleuropas. Band 3: Adephaga 2, Palpicornia, Histeroidea, Staphylinoidea 1. 365 p. Krefeld.

Hennig (W.). 1966. - Phylogenetic systematics. 263 p. Univ. Illinois Press, Urbana.

IENISTEA (M. A.). 1968. - Die Hydraeniden Rumäniens (Coleoptera Hydraenidae). Trav. Mus. Hist. nat. Gr. Antipa, $8: 759-795$.

Janssens (E.). 1965. - Les Hydraena de l'Egéide. Mém. Acad. r. Belg., Cl. Sc. $2^{\circ}$ sér., 16 (4) : 1-126.

Janssens (E.). 1967 a. - Une espèce nouvelle d'Hydraena du Tyrol: H. (Haenydra) tyrolensis n. sp. Bull. Inst. r. Sci. nat. Belg., 43 (1) : 1-3.

JANSSENS (E.). 1967 b. - Sur quelques Hydraenidae de la faune australe. Bull. Inst. r. Sci. nat. Belg., 43 (11): 1-13.

JANSSENS (E.). 1972. - Essai sur la systématique des Hydraena des régions intertropicales. Bull. Annls Soc. r. ent. Belg., 108: 253-261.

Kuwert (A.). 1890. - Bestimmungstabellen der Parniden Europas, der Mittelmeerfauna sowie der angrenzenden Gebiete (Col.). Verh. zool.bot. Ges. Wien, $40: 15-54$.

OlMI (M.). 1970. - Su alcuni Driopidi ed Elmintidi di Spagna (Coleoptera Dryopoidea). Memorie Mus. civ. Stor. nat. Verona, $17: 147-150$.

ORChyMONT (A. d'). 1931. - Hydraena nouvelles récoltées aux Balkans. Diagnoses préliminaires. Bull. Annls Soc. r. ent. Belg., 71 : 28-34. 
Orchymont (A. d'). 1936 a. - Inventa entomologica itineris Hispanici et Maroccani, quod a. 1926 fecerunt Harald et Håkan Lindberg. XXII Palpicornia. Commentat. biol., 5 (1): 1-22.

Orchymont (A. d'). 1936 b. - Les Hydraena de la Péninsule Ibérique (en annexe, synonymie de deux formes méditerranéennes). Mém. Mus. r. Hist. nat. Belg., 2 $2^{\mathrm{e}}$ sér., $6: 1-48$.

ORCHYMONT (A. d'). 1938. - Hydraena dentipes Germar en Belgique et en France (Coleoptera Palpicornia). Sa distribution géographique en rapport avec la nature minéralogique du sol et avec les phénomènes glaciaires quaternaires. Bull. Mus. r. Hist. nat. Belg., 14 (2) : 1-31.

Orchymont (A. d'). 1940. - Les Palpicornia des îles atlantiques. Mém. Mus. r. Hist. nat. Belg., $2^{\mathrm{c}}$ sér., 20 : 1-86.

Orchymont (A. d'). 1943. - Hydraena (Haenydra) nouvelles. Bull. Mus. r. Hist. nat. Belg., 19 (40): 1-8.

Orchymont (A. d'). 1944. - Le phylum Hydraena (s. str.) pulchella-pygmaea (Coleoptera Palpicornia Hydraenidae). Bull. Mus. r. Hist. nat. Belg., 20 (20): : 1-8.

PRETNER (E.). 1970. - Hydraena (subg. Haenydra) in Jugoslawien (Coleoptera : Palpicornia, Hydraenidae). Razpr. slov. Akad. Znan. Umet. Cl. IV Hist. nat. Medic., 13 : 115-151. 